\title{
LabVIEW y la instrumentación virtual aplicados a la docencia y la investigación en ciencias básicas
}

\author{
César Quiñones ${ }^{\star}$, Miguel Bernal ${ }^{\star \star}$ \\ Politécnico Grancolombiano
}

FECHA DE RECEPCión: Diciembre 1 DE 2010

FECha de APRobación: MARzo 23 DE 2011

\begin{abstract}
Resumen Este artículo muestra las principales características del lenguaje de programación gráfica LabVIEW y la instrumentación virtual. Muestra las características más importantes de este tipo de instrumentación, las ventajas y posibilidades que ofrece en el entorno de las ciencias básicas como una herramienta para la docencia y la investigación. Es un articulo de divulgación que busca servir de material introductorio para profesores y estudiantes que quieran trabajar con este tipo de tecnología y buscan entender los conceptos básicos antes de enfrentarse a un manual, un libro de texto o un curso virtual.
\end{abstract}

\begin{abstract}
This article shows the main characteristics of the graphical programming language LabVIEW as well as virtual instrumentation. It describes the most important characteristics of this type of instrumentation. Also, the advantages and possibilities it offers within the environment of basic sciences as a tool for teachers and researchers. This is an informative article that can be used as introductory material for teachers and students who want to work with this type of technology and understand its basic concepts before dealing with manuals, text books, or virtual courses.
\end{abstract}

Palabras Clave: LabVIEW, instrumentación virtual.

Keywords: LabVIEW, virtual instrumentation.

* Ph.D Química. Universidad Nacional de Colombia. caquinones@poli.edu.co

** Licenciado en Física, Universidad Pedagógica Nacional UPN. Profesor de planta del Politécnico Grancolombiano. Campos de interés: física computacional, atómica y de la materia condensada. mabernal@poli.edu.co 


\section{Introducción}

LabVIEW 1.0 fue lanzado al mercado en 1986 por la compañía National Instruments. Desde entonces integró características importantes que aun hoy después de dos décadas de desarrollo y mejoras siguen siendo el núcleo fundamental de este entorno de programación. El remplazo de líneas de texto por iconos gráficos y la escritura de código por el enlace de estos iconos ha logrado disminuir mucho los tiempos de desarrollo que se requieren para crear un instrumento virtual. Originalmente creado para computadores mac, hoy este tipo de instrumentación abarca una gran variedad de sistemas operativos desde Windows hasta sistemas en tiempo real.

La instrumentación virtual integra este lenguaje de programación con hardware especializado para crear soluciones que permiten adquirir, analizar y presentar datos en laboratorios de ciencia básica e ingeniería aprovechando al máximo las capacidades de calculo y comunicación de los computadores modernos.

El éxito de este tipo de instrumentación ha sido muy grande y puede atribuirse a la facilidad de uso del entorno gráfico de programación y a los dispositivos de hardware auto configurables plug and play que permiten a profesionales de muchas áreas trabajar en monitoreo y control de sistemas sin ser especialistas en software, programación o electrónica. Otro parámetro importante ha sido la capacidad de integración con áreas específicas del conocimiento por medio del desarrollo de Tools Kits especializados que permiten crear aplicaciones avanzadas utilizando herramientas prediseñadas [1].

\section{Presentación del entorno LabVIEW}

Un programa desarrollado en LabVIEW es llamado un instrumento virtual y tiene la extensión .VI. Está compuesto por un panel frontal y un diagrama de bloques. El primero es la interface humano máquina en la cual se encuentran controles e indicadores que permiten al usuario interactuar con el instrumento virtual. Los controles e indicadores simulan la apariencia de un instrumento real. De esta forma el operador puede manipular las variables del programa sin tener ningún conocimiento en programación. Este panel frontal ha sido uno de los grandes logros de LabVIEW ya que es fácilmente entendido y manejado por científicos e ingenieros de todas las ramas. Todos los controles pueden ser modificados por el usuario en posición, tamaño y color. Además, cuenta con algunos paquetes adicionales altamente especializados que permiten colocar controles e indicadores propios de campos muy específicos, algunos de esos paquetes son por ejemplo el DSSC (Data Login Sytem Control) que facilita la adquisición de datos y el control de instrumentos industriales y el Multisim para el diseño de aplicaciones con circuitos. Todos los controles e indicadores se encuentran en la paleta de controles que esta disponible solo para el panel frontal, esta paleta se muestra en la figura 1 .

En el diagrama de bloques se encuentra el código de programación el cual esta basado en iconos que se conectan para establecer un flujo de datos, cada control 
e indicador del panel frontal tiene un terminal que lo representa en el diagrama de bloques y desde o hacia este terminal viajan datos a través de alambres de conexión. Todas las herramientas y funciones se obtienen de la paleta de funciones que esta disponible en el diagrama de bloques, las diferentes funciones están agrupadas en sub paletas según su utilidad. Existen subpaletas tales como instrument i/o (para comunicación con instrumentos externos), Mathematics (para cálculos estadísticos, ajuste de datos o trabajo en álgebra lineal) y signal processing para el tratamiento de señales, entre muchas otras. De esta manera y conociendo la organización de las funciones dentro de cada una de las subpaletas, la programación puede hacerse de manera totalmente intuitiva. Aunque LabVIEW puede entender subrutinas basadas en lenguajes de texto importadas desde otros lenguajes de programación, los instrumentos virtuales pueden ser hechos en su totalidad colocando iconos gráficos, que representan funciones, dentro de estructuras de ejecución.

La figura 1 muestra un instrumento virtual sencillo para generar ondas y mostrarlas en el panel frontal. El panel frontal tiene un indicador (la gráfica) y tres controles (selector signal type, frecuencia y control start/stop). En el diagrama de bloques se observan los terminales de los controles e indicadores que se han colocado en el panel frontal, la estructura de programación while que ejecuta las rutinas que se encuentran dentro de ella hasta que el control stop sea activado y la función generar de señales, todas ellas conectadas por medio de alambres cuyos diferentes colores indican los diferentes tipos de datos que viajan a través de ellos.

\section{Instrumentación virtual}

La instrumentación virtual nace de la utilización del computador como una herramienta de medida y análisis de datos en el laboratorio. Aunque los programas que se desarrollan en LabVIEW se llaman instrumentos virtuales, en su forma mas general un instrumento virtual es la unión de un computador con el software y el hardware especializado que le permiten realizar las mismas funciones que un instrumento real. Estas funciones son adquirir, almacenar, analizar, mostrar y comunicar datos y tomar acciones de control sobre determinado proceso cuando así se requiera. Un instrumento virtual no es una simulación, el termino virtual se refiere a que el instrumento no existe como un instrumento tradicional por separado si no como parte de un sistema basado en software. Software que, en la pantalla del computador simula la apariencia de un instrumento real [2].

Las ventajas de utilizar instrumentación virtual son esencialmente todas aquellas que se derivan de la utilización de un computador y son entre otras: alta velocidad de procesamiento de datos, capacidad prácticamente ilimitada de almacenamiento de datos, comunicación de datos a diferentes niveles y altas velocidades, toma de decisiones según argumentos lógicos preestablecidos y capacidad comunicación con instrumentos externos o interfases de adquisición de datos por diferentes tipos de interfaces o buses disponibles. 


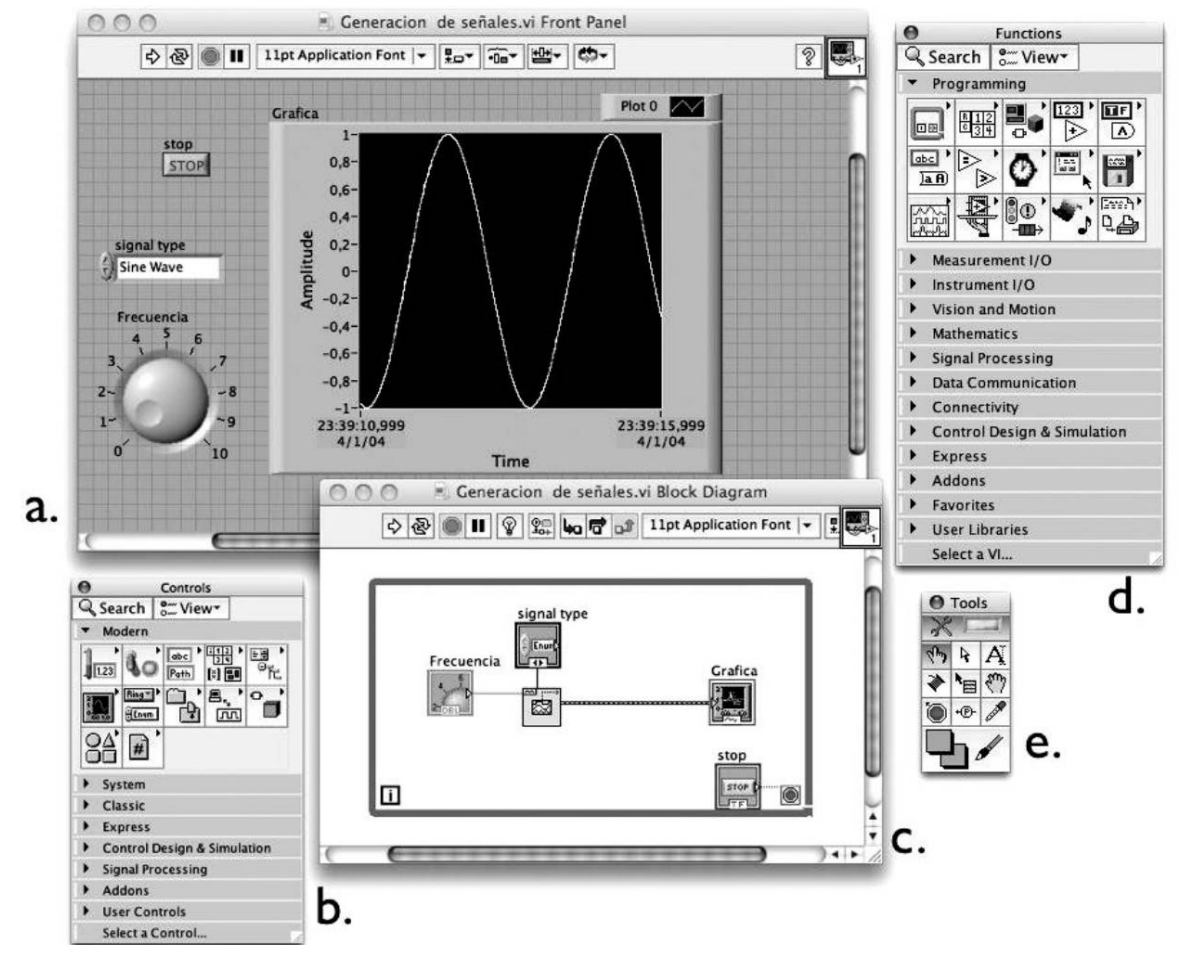

Figura 1. Estructura de un instrumento virtual. a. Panel frontal con controles e indicadores. b. Paleta de controles. c. Diagrama de bloques con funciones y terminales d. Paleta de funciones e. Paleta de herramientas.

Un instrumento virtual es un instrumento de medida de arquitectura abierta lo que permite que pueda ser fácilmente escalable en caso de que se requiera aumentar el tipo o clase de señales que se están monitoreando o controlando. Es además reconfigurable y puede ser diseñado de manera tal que se acople a los requerimientos de un usuario particular.

Cuando nos referimos a hardware especializado hacemos referencia específicamente a dos clases de hardware. Primero, diferentes instrumentos de medida disponibles en un laboratorio como osciloscopios, multímetros o medidores de pH que cuentan con interfaces de comunicación que les permiten ser conectados al computador y controlados desde LabVIEW. Segundo, interfaces de conversión análogo/digital o digital/análogo de National Instruments u otra compañía de instrumentación. LabVIEW cuenta con una gran cantidad de drivers para diferentes instrumentos de laboratorio e interfaces de adquisición de datos de otros fabricantes disponibles de forma gratuita en el sitio Web de la compañía. De esta manera las posibilidades para escoger o acoplar hardware ya existente en el laboratorio son muy amplias. 


\section{La instrumentación virtual como herramienta docente}

El concepto sobre el cual se desarrollo LabVIEW permite que el tiempo necesario para el desarrollo de aplicaciones sea muy corto si se compara con otros lenguajes de programación gracias a que es un lenguaje grafico e intuitivo. Estas características hacen posible la inclusión del estudiante en el desarrollo de sistemas de adquisición de datos en laboratorios de química o física experimental, por tal motivo LabVIEW ha sido incluido dentro de currículo de varias carreras científicas y de ingeniería e incorporado como un a herramienta docente en laboratorios de instrumentación, análisis y adquisición de datos [3], [4], [5].

Dentro de un laboratorio de docencia LabVIEW puede ser utilizado para hacer adquisición de datos [6], [7], establecer correlaciones [8, generar reportes en una practica de laboratorio los cuales pueden asemejarse a un informe de laboratorio [9], hacer simulaciones [10], mejorar y automatizar medidas de laboratorio que previamente se realizaban manualmente [11, mejorar el proceso de enseñanza [12], o diseñar sistemas de adquisición remotos [13].

La instrumentación virtual es ampliamente utilizada en la industria en la actualidad, razón por la cual trabajar con este tipo de instrumentación tiene como valor agregado el entrenamiento del estudiante con herramientas que encontrara en su entorno profesional. Las estructuras de programación en LabVIEW permiten que el estudiante tenga una idea clara y jerarquizada de los procesos de adquisición, análisis y presentación y comunicación de datos. El trabajo con instrumentación virtual en el laboratorio ha demostrado que cuando el estudiante es involucrado en el proceso de diseño y montaje de un sistema de monitoreo o control de alguna variable física o química, entiende mas fácilmente el fenómeno bajo estudio y puede proponer y comprobar hipótesis relacionadas con el fenómeno bajo estudio.

\section{La instrumentación virtual como herramienta investigativa}

La mayoría de las interfaces de adquisición de datos son multipropósito lo cual hace de los instrumentos virtuales instrumentos muy versátiles. Existen muchos ejemplos de instrumentación virtual avanzada aplicada a laboratorios de investigación [14, [15], [16], 17] y en todos ellos el computador juega un papel fundamental como herramienta de análisis y almacenamiento de datos. Las variables más comunes en este tipo experimentos son temperatura, nivel, presión, voltaje y resistencia. Otras que pueden monitorearse son $\mathrm{pH}$, concentración de especies químicas en solución, flujo, aceleración y corriente eléctrica y cualquier otra en la que se cuente con un sensor adecuado que produzca una señal analógica o digital que pueda ser leída por una interfase de datos que se conecte al computador.

Cuando los experimentos así lo requieran puede contarse con interfaces de especificaciones muy altas ya sea alta velocidad de adquisición, amplificación o filtrado de señales o respuesta en tiempo real. En general puede decirse que un sistema monitoreo y control basado en instrumentación virtual puede tomar 
decisiones acerca del almacenamiento, muestreo, análisis y control de variables en experimento dado, facilitado de esta manera la labor investigativa ya que libera tiempo del investigador, suministra mejores datos y permite hacer un análisis detallado de los datos.

\section{Conclusión}

LabVIEW y la instrumentación virtual basada en computadores son herramientas poderosas en la academia a nivel investigativo y docente. Este lenguaje de programación puede ser ejecutado sobre diferentes plataformas y proporciona acceso a la capacidad de almacenamiento, análisis y comunicación propia de los computadores modernos. Las aplicaciones son tan diversas como numerosas y pueden dar una idea clara de la versatilidad de las posibles aplicaciones que pueden lograrse. Libros especializados, blogs, foros y cursos libres disponibles en internet son algunas de las múltiples posibilidades que existen para aprender a manejar este lenguaje de programación. El logro más grande de este tipo de programación e instrumentación es poner al alcance de científicos, ingenieros docentes e investigadores una gran cantidad de nuevas posibilidades antes reservadas solamente a programadores e instrumentadores expertos.

\section{Referencias}

1. Lajara, J., Pelegri, J.: LabVIEW: Entorno gráfico de programación. Alfaomega, Mexico D. F. (2007)

2. Lázaro, A., Río, J.: LabVIEW: Programación grafica para el control de instrumentación. Thomson, Madrid. (2005)

3. Mason, D. S.: Preparing the Professional Chemist . J. Chem. Educ., Vol. 80 No. 6. (2003)

4. Drew, S. M.: Integration of National Instruments LabVIEW Software in to the Chemistry Curriculum. J. Chem. Educ. Vol. 73 No. 12. (1996)

5. Gostowski, R.: Teaching Analytical Instrument Design with LabVIEW 1, J. Chem. Educ. Vol. 73 No. (1996)

6. Muyskens, M. A., Glass, S. V., Wietsma, T. W., Gray T. M.: Data Acquisition in the Chemistry Laboratory Using LabVIEW Software, J. Chem. Educ. Vol. 73 No. 12. (1996)

7. Ogren, P. J., Jones, T. P.: Laboratory Interfacing Using the LabVIEW Software Package, J. Chem. Educ.Vol. 73 No 12. (1996)

8. Hovick, J. W., Murphy, M., Poler J. C.: An Introduction to Correlation Techniques, "Audibilization" in the Chemistry Laboratory: for Data Extraction, J. Chem. Educ., Vol. 84 No. 8. (2007)

9. Belletti, A., Borromei, R., Ingletto, G.: EQVAPSIM: A Vapor-Liquid Equilibria of Binary Systems, Computer Simulation by LabVIEW, J. Chem. Educ., Vol. 85 No. 6. (2008)

10. Belletti, A., Borromei, R., Ingletto, G.: Teaching Physical Chemistry Experiments with a Computer Simulation by LabVIEW, J. Chem. Educ., Vol. 83 No. 9. (2006)

11. Urian, R. C., Khundkar, L. R.: A Diode-Laser-Based Automated Timing Interface for Rapid Measurement of Liquid Viscosity, Department of Chemistry, Northeastern University, Boston, J. Chem. Educ., Vol. 75 No. 9. (1998) 
12. Bell, M. A., Gladwin, R. P., Drury, T. A.: Computer-assisted learning. Implementing CAL in Chemistry, J. Chem. Educ., Vol. 75 No. 6. (1998)

13. Baran, J., Currie, R.: Remote Instrumentation for the Teaching Laboratory Chemical Technology, J. Chem. Educ., Vol. 81 No. 12. (2004)

14. Malina, E. G., Nakhleh, M. B.: How Students Use Scientific Instruments to Create Understanding: CCD Spectrophotometers, J. Chem. Educ., Vol. 80 No. 6. (2003)

15. Martinez, L. M., Videa, M., Mederos, F., Mesquita, J.: Differential Thermal Analysis Device for Teaching and Research, J. Chem. Educ.,Vol. 84 No. 7. (2007)

16. Spanoghe, P., Cocquyt, J., Van der Meeren, P.: A Low-Cost Dynamic Surface Tension Meter with a LabVIEW Interface and Its Usefulness in Understanding Foam Formation, J. Chem. Educ. Vol 78, No 3. (2001)

17. Jensen, M. B.: Integrating HPLC and Electrochemistry: A LabVIEW - Based Pulsed Amperometric Detection System, Vol. 79 No. 3. (2002)

18. Bailey, R. A., Desai, S. B., Hepfinger, N. F., Hollinger, H. B., Locke, P. S., Miller, K. J.: Simultaneous Recording of Multiple Cooling Curves, J. Chem. Educ., Vol 74, No 6. (1997) 